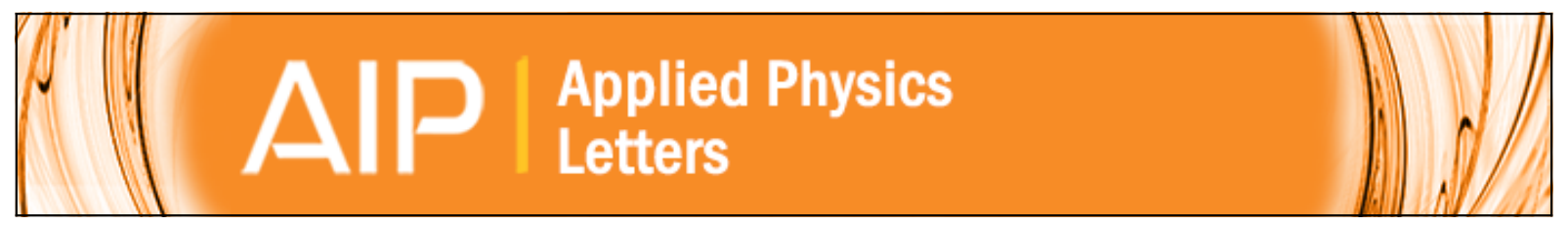

Pinned synthetic ferrimagnets with perpendicular anisotropy and tuneable exchange bias

J. Sort, B. Rodmacq, S. Auffret, and B. Dieny

Citation: Applied Physics Letters 83, 1800 (2003); doi: 10.1063/1.1606495

View online: http://dx.doi.org/10.1063/1.1606495

View Table of Contents: http://scitation.aip.org/content/aip/journal/apl/83/9?ver=pdfcov

Published by the AIP Publishing

\title{
AAlP
}

\section{Create a profile.




\title{
Pinned synthetic ferrimagnets with perpendicular anisotropy and tuneable exchange bias
}

\author{
J. Sort, ${ }^{\text {a) }}$ B. Rodmacq, S. Auffret, and B. Dieny \\ SPINTEC, Département de Recherche Fondamentale sur la Matière Condensée, CEA/Grenoble, \\ 38054 Grenoble Cedex 9, France
}

(Received 7 May 2003; accepted 2 July 2003)

\begin{abstract}
Pinned synthetic ferrimagnets (syFerri) with perpendicular-to-plane magnetic anisotropy, of the form $\mathrm{AP}_{1} / \mathrm{Ru} / \mathrm{AP}_{2} / \mathrm{FeMn}$ [where $\mathrm{AP}_{1}$ and $\mathrm{AP}_{2}$ are $(\mathrm{Co} / \mathrm{Pt})$ multilayers], have been prepared and characterized. The magnitudes of the exchange bias fields of both $\mathrm{AP}_{1}$ and $\mathrm{AP}_{2}$ can be tuned at room temperature by simply varying the relative number of $(\mathrm{Co} / \mathrm{Pt})$ repeats in each multilayer. This effect can be quantitatively interpreted by considering the different energy contributions involved during magnetization reversal. Moreover, from the values of these fields, the characteristic parameters of the system (i.e., coupling strength through the $\mathrm{Ru}$ and $\mathrm{AP}_{2} / \mathrm{FeMn}$ pinning energy), can be evaluated. Interestingly, an extended plateau with a virtually constant magnetization is observed around zero field when the number of $\mathrm{Co} / \mathrm{Pt}$ repeats in $\mathrm{AP}_{1}$ is equal or larger than in $\mathrm{AP}_{2}$. This is very appealing for field sensor or memories applications using spin valves or tunnel junctions with perpendicular anisotropy, since it offers a large dynamic range over which the magnetic configuration of the syFerri remains stable. (C) 2003 American Institute of Physics.
\end{abstract}

[DOI: $10.1063 / 1.1606495]$

Recently, in spin-valve based read heads for high density magnetic recording media, ${ }^{1}$ the replacement of the traditional antiferromagnetic (AFM) exchange layer by synthetic antiferromagnets (syAFM) has been found to be very appealing, since syAFM considerably enhance the thermal and magnetic stability of these sensors and reduce the magnetostatic field created by the pinned layer on the free layer in submicronic devices. ${ }^{2}$ Typically, a syAFM consists of two ferromagnetic (FM) layers separated by a nonmagnetic spacer, such as $\mathrm{Ru}(6-10 \AA$ thick), which couples them antiferromagnetically due to the Ruderman-Kittel-(Kasuya)-Yosida $[\mathrm{RK}(\mathrm{K}) \mathrm{Y}]$ interaction. $^{3}$

All syAFM-based spin valves elaborated so far exhibit an in-plane magnetic anisotropy. Just recently, spin valves and tunnel junctions with perpendicular-to-plane anisotropy have also been developed. ${ }^{4}$ However, they do not make use of syAFM. Studies on syAFM-like systems with perpendicular anisotropy are very scarce ${ }^{5}$ and the effect of varying the relative thickness of the two FM layers in contact with $\mathrm{Ru}$ has only been systematically investigated in in-plane syAFM. ${ }^{6}$ Nevertheless, a few studies on the physics of antiferromagnetically coupled multilayers with perpendicular anisotropy have been reported. ${ }^{7,8}$

In this letter, we demonstrate the feasability to grow pinned synthetic ferrimagnets (syFerri) with perpendicular anisotropy, exhibiting tuneable exchange bias at room temperature. The systems consist of two antiferromagnetically coupled (Pt/Co) multilayers (ML), denoted as $\mathrm{AP}_{1}$ and $\mathrm{AP}_{2}$, in which $\mathrm{AP}_{2}$ is, in turn, exchange biased with an $\mathrm{AFM} .{ }^{9}$ The switching fields of both $\mathrm{AP}_{1}$ and $\mathrm{AP}_{2}$ can be easily tuned by simply varying the relative number of $(\mathrm{Pt} / \mathrm{Co})$ repeats in each multilayer.

A series of pinned synthetic ferrimagnets with the com-

${ }^{a)}$ Electronic mail: sort@drfmc.ceng.cea.fr position Pt $(50 \AA) /[\mathrm{Co}(5 \AA) / \operatorname{Pt}(20 \AA)]_{n} / \operatorname{Co}(5 \AA) / \operatorname{Ru}(7 \AA) /$ $[\mathrm{Co}(5 \AA) / \operatorname{Pt}(20 \AA)]_{3} / \operatorname{Co}(5 \AA) / \operatorname{FeMn}(130 \AA) / \operatorname{Pt}(20 \AA)$, i.e., $\mathrm{AP}_{1} / \mathrm{Ru} / \mathrm{AP}_{2} / \mathrm{FeMn}$, with $n=1,2,3,4$, and 5, were deposited onto thermally oxidized $\mathrm{Si}$ substrates by de magnetron sputtering. The $\mathrm{AP}_{1}$ multilayer contains a variable number of Co layers, i.e., $n_{\mathrm{AP} 1}=n+1$, whereas $\mathrm{AP}_{2}$ contains always 4 Co layers. All depositions were performed at room temperature. A stray field perpendicular to plane is known to exist over the substrate area in the sputtering unit. Hysteresis loops of as-deposited samples were recorded using extraordinary Hall effect, with magnetic field applied perpendicular to the film direction. ${ }^{10}$

Figure 1 shows the hysteresis loops of the pinned syFerri with $n_{\mathrm{AP} 1}=2,3,4$, and 6 . The magnetic configurations of $\mathrm{AP}_{1}$ and $\mathrm{AP}_{2}$ multilayers are indicated by the thin and thick arrows, respectively. It can be seen that when the field is reduced from positive to negative saturation, the magnetization of $\mathrm{AP}_{1}\left(M_{\mathrm{AP} 1}\right)$ always switches before that of $\mathrm{AP}_{2}$, $M_{\mathrm{AP} 2}$, at a positive field denoted by $H_{E, \mathrm{AP} 1}^{+}$. A broad plateau is then observed around zero field, over which the two multilayers are in antiparallel magnetic configuration. When the field is further reduced, the magnetization of the $\mathrm{AP}_{2}$ layer $\left(M_{\mathrm{AP} 2}\right)$ finally switches at a negative field $H_{E, \mathrm{AP} 2}^{+}$. Interestingly, for $n_{\mathrm{AP} 1}=2$, the switching of $M_{\mathrm{AP} 2}$ is accompanied by a backswitching (i.e., spin-flip) of $M_{\mathrm{AP} 1}$, due to the antiferromagnetic $\mathrm{RK}(\mathrm{K}) \mathrm{Y}$ interaction through Ru. Finally, $M_{\mathrm{AP} 1}$ switches down again at a slightly larger negative field.

When increasing the field from negative to positive saturation, significant differences in the magnetization reversal process are observed depending on the value of $n_{\mathrm{AP} 1}$.

(1) For $n_{\mathrm{AP} 1}=2$ and 3, $M_{\mathrm{AP} 1}$ switches first at a characteristic negative field $H_{E, \mathrm{AP} 1}^{-}$. Note that since the energy balance which determines this switching field is exactly the same as the one which determines the switching field of $M_{\mathrm{AP} 1}$ at decreasing field from positive saturation, the rela- 

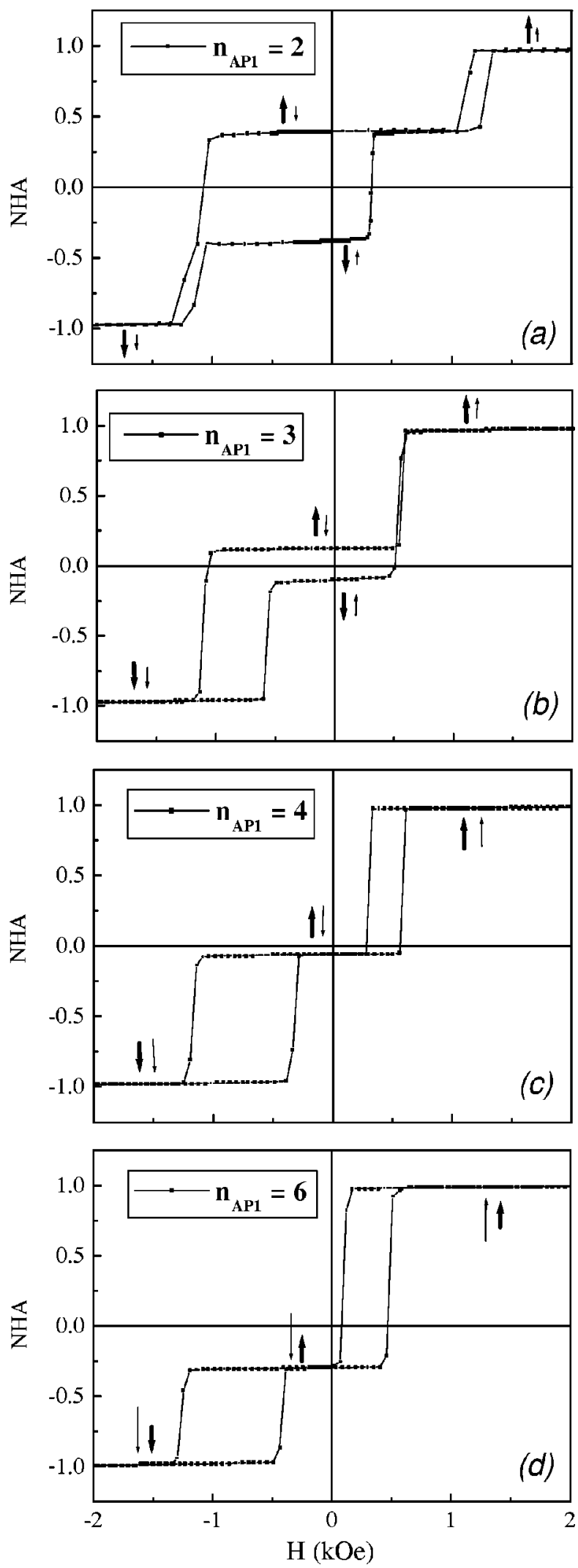

FIG. 1. Normalized Hall amplitude hysteresis loops of syFerri of the form $\mathrm{AP}_{1} / \mathrm{Ru} / \mathrm{AP}_{2} / \mathrm{FeMn}$, with $n_{\mathrm{AP} 1}=2,3,4$, and 6 (where $n_{\mathrm{AP} 1}$ is the number of Co layers in the $\mathrm{AP}_{1}$ multilayer). The thin and thick arrows indicate the magnetization state of multilayers $\mathrm{AP}_{1}$ and $\mathrm{AP}_{2}$ in the syFerri, respectively.

tionship $H_{E, \mathrm{AP} 1}^{-}=-H_{E, \mathrm{AP} 1}^{+}$is observed. As the field is further increased, $M_{\mathrm{AP} 2}$ switches. In the case $n_{\mathrm{AP} 1}=2$, this switching is again accompanied by a backswitching of $M_{\mathrm{APl}}$. In this case, another transition occurs at a larger posi-

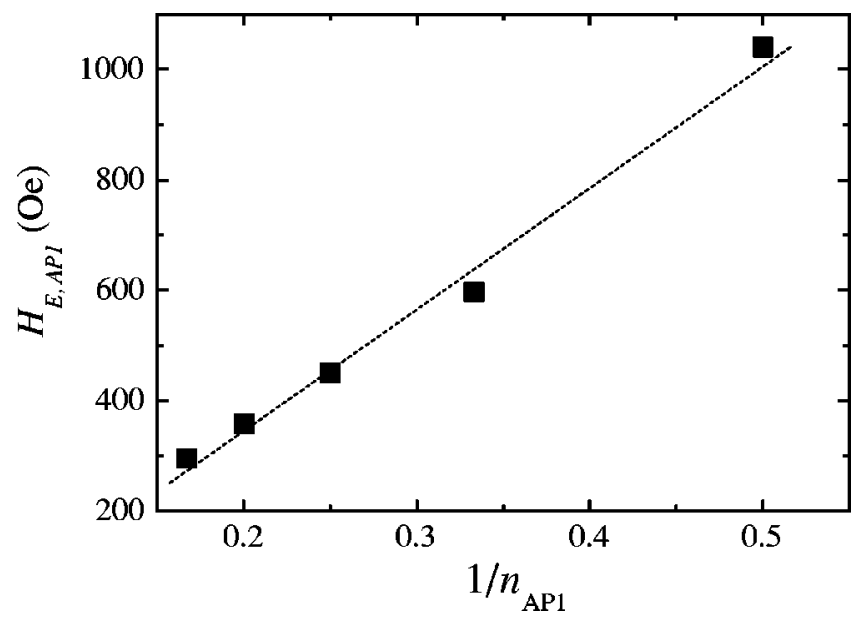

FIG. 2. Dependence of the exchange bias field of $\mathrm{AP}_{1} 1 / n_{\mathrm{AP} 1}$, where $n_{\mathrm{AP} 1}$ designates the number of Co layers in $\mathrm{AP}_{1}$.

tive field corresponding to the reversal of $M_{\mathrm{AP} 1}$. Note that the backswitching of $M_{\mathrm{AP} 1}$ is not observed for $n_{\mathrm{AP} 1}=3$ because it would cost too much Zeeman energy.

(2) For $n_{\mathrm{AP} 1} \geqslant 4, M_{\mathrm{AP} 2}$ switches first. This results in an extended plateau with constant magnetization around zero field. We point out that in these cases, there is only one stable remanent state of the system in zero field. This is a situation of practical interest for device applications in order to use such a synthetic pinned layer as a reference layer in spin valves or tunnel junctions with perpendicular magnetization. At a larger positive field, the magnetization $M_{\mathrm{AP} 1}$ reverses towards positive saturation.

The differences in the magnetization curves of the different systems can be understood as the result, in each syFerri, of an interplay between the Zeeman energy, the exchange energy across the Ru spacer and the pinning energy with the FeMn. The purpose of the following calculations is to relate the values of the $\mathrm{AP}_{1}$ and $\mathrm{AP}_{2}$ switching fields with the coupling energy per unit area through the $\mathrm{Ru}$ spacer $\left(A_{\mathrm{Ru}}\right)$ and the coupling energy per unit area with the antiferromagnetic layer $\left(A_{\text {pinning }}\right)$. These calculations assume that the system is always able to reach its lowest energy minimum, i.e., as a first step, we do not try to describe the irreversibility associated with the switching of $M_{\mathrm{AP} 1}$ and $M_{\mathrm{AP} 2}$.

Let us first consider the switching of $\mathrm{AP}_{1}$ when decreasing field from positive saturation. This switching occurs at a positive field when the gain in coupling energy due to the antiferromagnetic coupling through the $\mathrm{Ru}$ spacer balances the cost in Zeeman energy due to the negative orientation of $M_{\mathrm{AP} 1}$ in the positive field. If $t_{\mathrm{Co}}$ is the thickness of the individual Co layers and $\mathrm{Ms}_{\mathrm{Co}}$ its saturation magnetization, then the bias field of $\mathrm{AP}_{1}, H_{E, \mathrm{AP} 1}$ (evaluated as the average of the two switching fields in the $\mathrm{AP}_{1}$ minor loop), is given by:

$$
n_{\mathrm{AP} 1} \mathrm{Ms}_{\mathrm{Co}} t_{\mathrm{Co}} H_{E, \mathrm{AP} 1}=\mathrm{A}_{\mathrm{Ru}} \text {. }
$$

This expression predicts a linear variation of $H_{E, \mathrm{AP} 1}$ vs $1 / n_{\mathrm{AP} 1}$, provided that $A_{\mathrm{Ru}}$ is constant. If one plots $H_{E, \mathrm{AP} 1}$ as a function of $1 / n_{\mathrm{AP} 1}$, a linear dependence of $H_{E, \mathrm{AP} 1}$ is experimentally observed, as shown in Fig. 2. Assuming $\mathrm{Ms}_{\mathrm{Co}}$ $=1450 \mathrm{emu} / \mathrm{cm}^{3}$ (bulk value), the slope of $H_{E, \mathrm{AP} 1}\left(1 / n_{\mathrm{AP} 1}\right)$ in Fig. 2 allows us to determine $A_{\mathrm{Ru}}$. One obtains $A_{\mathrm{Ru}}$ $=0.134 \mathrm{erg} / \mathrm{cm}^{2}$. Note that the possibility to tune perpen- 
dicular exchange bias by changing the number of $\mathrm{Co} / \mathrm{Pt}$ repeats has already been reported in the literature. ${ }^{11-13}$ The coercivity of $\mathrm{AP}_{1}$ can be also easily determined from the $\mathrm{AP}_{1}$ minor loops. The coercive field increases with $n_{\mathrm{AP} 1}$, as commonly observed in $(\mathrm{Pt} / \mathrm{Co})$ multilayers, from 95 Oe for $n_{\mathrm{AP} 1}=2$ to 200 Oe for $n_{\mathrm{AP} 1}=6$. This is due to an increase of the overall perpendicular anisotropy in $\mathrm{AP}_{1}$ with the number of $\mathrm{Pt} / \mathrm{Co}$ interfaces, as well as to the increase of structural defects, which may act as pinning centers for domain wall propagation.

The switching of $M_{\mathrm{AP} 2}$ at negative fields occurs when the Zeeman energy brought by the applied field exceeds the cost in energy to overcome both the antiferromagnetic coupling through the Ru spacer and the exchange bias energy with FeMn. The associated coercivity can be clearly seen for $n_{\mathrm{AP} 1}=4$ and 6 from the width of the $\mathrm{AP}_{2}$ minor loops [Figs. $1(\mathrm{c})$ and $1(\mathrm{~d})]$. Note that the coercivity of $\mathrm{AP}_{2}$ is larger than for $\mathrm{AP}_{1}$ for the same number of repeats due to the exchange biasing of $\mathrm{AP}_{2}$ with FeMn. ${ }^{9}$ The bias field of $\mathrm{AP}_{2}, H_{E, \mathrm{AP} 2}$, can be evaluated by considering the shift of the $\mathrm{AP}_{2}$ minor loops for $n_{\mathrm{AP} 1}=4$ and 6 . One finds $H_{E, \mathrm{AP} 2}$ to be around 810 Oe. $H_{E, \mathrm{AP} 2}$ is then related to the coupling constants by the expression

$$
n_{\mathrm{AP} 2} \mathrm{Ms}_{\mathrm{Co}} t_{\mathrm{Co}} H_{E, \mathrm{AP} 2}=-\left(\mathrm{A}_{\mathrm{Ru}}+\mathrm{A}_{\text {pinning }}\right) .
$$

This relationship allows to calculate $A_{\text {pinning }}$ from the knowledge of $H_{E, \mathrm{AP} 2}$. Namely $A_{\text {pinning }}=0.068 \mathrm{erg} / \mathrm{cm}^{2}$.

The values of $H_{E, \mathrm{AP} 2}$ in the syFerri are significantly larger than the ones for Pt/Co ML exchange biased with FeMn (i.e., about 810 Oe, compared 150 Oe in simple ML + FeMn structures).

It is also worthwhile to illustrate why the layers switch the way they do and not in the reverse order. Note that, when decreasing field from positive saturation, if $M_{\mathrm{AP} 2}$ switched before $M_{\mathrm{AP} 1}$, Eqs. (1) and (2) would no longer govern the reversal. Instead, the hypothetical bias field of $\mathrm{AP}_{2}$, $H_{E, \mathrm{AP} 2}($ hypo) would be given by the following equation:

$$
n_{\mathrm{AP} 2} \mathrm{Ms}_{\mathrm{Co}} t_{\mathrm{Co}} H_{E, \mathrm{AP} 2 \text { (hypo) }}=\left(A_{\mathrm{Ru}}-A_{\text {pinning }}\right) .
$$

Comparing the values of $H_{E, \mathrm{AP} 1}$ and $H_{E, \mathrm{AP} 2}$ (hypo) [Eqs. (1) and (3)], it is found that $M_{\mathrm{AP} 2}$ would reverse before $M_{\mathrm{AP} 1}$ only if

$$
\frac{A_{\mathrm{Ru}}-A_{\text {pinning }}}{n_{\mathrm{AP} 2}}>\frac{A_{\mathrm{Ru}}}{n_{\mathrm{AP} 1}} .
$$

Taking the previously determined values of $A_{\mathrm{Ru}}$ and $A_{\text {pinning }}$ one finds that Eq. (4) is never fulfilled, which explains why $M_{\mathrm{AP} 1}$ always switches first when the field is decreased from positive saturation.

Analogously, when the field is increased from negative saturation, it can be shown that $M_{\mathrm{AP} 2}$ reverses before $M_{\mathrm{AP} 1}$ only if

$$
\frac{A_{\mathrm{Ru}}+A_{\text {pinning }}}{n_{\mathrm{AP} 2}}>\frac{A_{\mathrm{Ru}}}{n_{\mathrm{AP} 1}} .
$$

Condition (5) is fulfilled for $n_{\mathrm{AP} 1}=3,4,5$, and 6 and, consistently, it is experimentally observed that $M_{\mathrm{AP} 2}$ reverses before $M_{\mathrm{AP} 1}$ for $n_{\mathrm{AP} 1}=4,5$, and 6. Similarly, Eq. (5) is not fulfilled for $n_{\mathrm{AP} 1}=2$ in agreement with the experimental observation that $M_{\mathrm{AP} 1}$ switches first. For $n_{\mathrm{AP} 1}=3$, although inequality (5) is fulfilled, $M_{\mathrm{AP} 1}$ switches before $M_{\mathrm{AP} 2}$ [see Fig. 1(c)]. This is because the coercive field in $\mathrm{AP}_{2}$ is always larger than in $\mathrm{AP}_{1}$.

In conclusion, it has been shown that pinned synthetic ferrimagnets with perpendicular anisotropy, can be prepared. The bias fields of both ML constituting the syFerri can be tuned at room temperature by varying the relative number of $\mathrm{Co} / \mathrm{Pt}$ repeats comprised in each ML and their values are determined by the exchange constants $A_{\mathrm{Ru}}$ and $A_{\text {pinning }}$. However, an extended plateau with constant magnetization around zero field and a single stable remanent state is obtained when the condition $n_{\mathrm{AP} 1}>A_{\mathrm{Ru}} /\left(A_{\mathrm{Ru}}+A_{\text {pinning }}\right) n_{\mathrm{AP} 2}$ is fulfilled. These properties are particularly useful to implement spin valves or tunnel junctions with perpendicular anisotropy and enhanced magnetic stability.

This work was supported by the European Community through the NEXBIAS Grant No. HPRN-CT-2002-00296. The authors acknowledge Josep Nogués for the critical reading of the manuscript.

${ }^{1}$ B. Dieny, V. S. Speriosu, S. S. P. Parkin, B. A. Gurney, D. R. Wilhoit, and D. Mauri, Phys. Rev. B 43, 1297 (1991).

${ }^{2}$ J. L. Leal and M. H. Kryder, J. Appl. Phys. 83, 3720 (1998); J. G. Zhu, IEEE Trans. Magn. 35, 6807 (1999).

${ }^{3}$ S. S. P. Parkin, N. More, and K. P. Roche, Phys. Rev. Lett. 64, 2304 (1990).

${ }^{4}$ F. Garcia, F. Fettar, S. Auffret, B. Rodmacq, and B. Dieny, J. Appl. Phys. 93, 8397 (2003).

${ }^{5}$ T. L. Kirk, O. Hellwig, and E. E. Fullerton, Phys. Rev. B 65, 224426 (2002).

${ }^{6}$ B. Dieny, M. Li, S. H. Liao, C. Horng, and K. Ju, J. Appl. Phys. 87, 3415 (2000); K. Y. Kim, S. H. Jang, K. H. Shin, H. J. Kim, and T. Kang, ibid. 89, 7612 (2001); K. Inomata, T. Nozaki, N. Tezuka, and S. Sugimoto, Appl. Phys. Lett. 81, 310 (2002).

${ }^{7}$ M. M. H. Willekens et al., Mater. Res. Soc. Symp. Proc. 313, 129 (1993).

${ }^{8}$ O. Hellwig, T. L. Kirk, J. B. Kortright, A. Beger, and E. E. Fullerton, Nat. Mater. 2, 112 (2003).

${ }^{9}$ For recent reviews on exchange bias, see J. Nogués and I. K. Schuller, J. Magn. Magn. Mater. 192, 203 (1999); A. E. Berkowitz and K. Takano, ibid. 200, 552 (1999); R. L. Stamps, J. Phys. D 33, R247 (2000); M. Kiwi, J. Magn. Magn. Mater. 234, 584 (2001).

${ }^{10}$ C. L. Canedy, X. W. Li, and G. Xiao, J. Appl. Phys. 81, 5367 (1997).

${ }^{11}$ S. Maat, K. Takano, S. S. P. Parkin, and E. E. Fullerton, Phys. Rev. Lett. 87, 087202 (2001).

${ }^{12}$ F. Garcia, J. Sort, B. Rodmacq, S. Auffret, and B. Dieny, Appl. Phys. Lett. (submitted).

${ }^{13}$ F. Garcia, J. Moritz, F. Ernult, S. Auffret, B. Rodmacq, B. Dieny, J. Camarero, Y. Pennec, S. Pizzini, and J. Vogel, IEEE Trans. Magn. 38, 2730 (2002). 